\title{
Adrenal artery pseudoaneurysm in pheochromocytoma presenting with catastrophic retroperitoneal haemorrhage
}

\author{
Santosh Kumar, MS, MCh, FRCS, ${ }^{*}$ Bhuvanesh Nanjappa, MS, MCh; ${ }^{*}$ Sathish Kumar, RM, MS, MCh, MRCS; \\ Seema Prasad, MD; ${ }^{\dagger}$ Arawat Pushkarna, MS,; Shrawan Kumar Singh, MS, MCh*
}

*Department of Urology, Postgraduate Institute of Medical Education and Research, Chandigarh, India; 'Department of Anesthesia, Postgraduate Institute of Medical Education and Research, Chandigarh, India; §Department of General Surgery, Postgraduate Institute of Medical Education and Research, Chandigarh, India

Cite as: Can Urol Assoc J 2013:E254-6. hittp://dx.doi.org/10.5489/cuai.541

\section{Abstract}

Spontaneous rupture of adrenal pheochromocytoma is an extremely rare condition which presents as an abdominal catastrophe. Unrecognized, this transformation can rapidly lead to death. We report a case of a 63-year-old male who presented with hemorrhagic shock secondary to ruptured adrenal pheochromocytoma. The clinical course is notable for immediate transarterial catheter embolization for control of bleeding, followed by optimization and elective adrenalectomy. High mortality is associated with an operative intervention in the face of an unrecognized pheochromocytoma. This reinforces the need for maintaining a high index of suspicion in the setting of a suprarenal mass despite hypotension.

\section{Introduction}

There are various causes of a retroperitoneal haemorrhage; these include bleeding from renal vessels, kidney, great vessels either spontaneously or after trauma. Rupture of a pheochromocytoma is rare and there are more than 50 reported cases. Massive release of catecholamines, as well as potential massive blood loss, makes it both an endocrinological and surgical emergency. Bleeding, heart failure, postoperative severe hypotension or pulmonary edema is the causes for perioperative mortality in these cases. ${ }^{1}$ Adrenalectomy is the treatment of choice after optimization. We report a case of retroperitoneal hemorrhage associated with pheochromocytoma.

\section{Case report}

A 63-year-old male presented to emergency with left loin pain and shock. He was clinically pale and his pulse was 120/ minute with blood pressure of $70 / 50 \mathrm{mmHg}$. Resuscitation was started and an urgent ultrasound (USG), done at his bedside, revealed a left suprarenal mass lesion. After he was stabilized, a contrast enhanced computed tomography (CECT) scan was done which revealed a large retroperitoneal hemorrhage above left kidney with intact vasculature of kidney (Fig. 1, Fig. 2). The patient was hemodynamically unstable and taken for transarterial embolization (TAE). It revealed an active bleeding at the left adrenal artery pseudoaneurysm, which was embolized with a coil. As soon as the embolization was done, his blood pressure rose to 200/110 mmHg for which nitroglycerin had to be started. A provisional diagnosis of pheochromocytoma was done and he started on alpha blockers. A repeat CECT was performed after 1 month which revealed a resolution of the hematoma and a left suprarenal mass. A metaiodobenzylguanidine (MIBG) scan revealed uptake in the left suprarenal mass lesion. However, 24-hour urinary metanephrines were within normal limits. Left adrenalectomy was performed after adequate preoperative preparation. Intraoperative hypertension during manipulation was managed by sodium nitroprusside. The patient's postoperative recovery was uneventful. The gross and cut-section images are shown in Fig. 3a and Fig. 3b. Histopathology was consistent with pheochromocytoma (Fig. 4a, Fig. 4b).

\section{Discussion}

Spontaneous rupture of pheochromocytoma has rarely been reported. ${ }^{1}$ Common presentations are headache, palpitations and flushing. Hypertension is the most common clinical sign. ${ }^{2}$ Retroperitoneal or tranperitoneal hemorrhage due to adrenal pheochromocytoma rupture is uncommon and many articles mention the clinical picture of hypertension in this scenario. ${ }^{1,3}$ We report a clinical paradox wherein a functional tumour presented with hypotension. Suspicion of pheochromocytoma is mandatory in situations like idiopathic retroperitoneal hemmorhage.

TAE of pheochromocytoma is not novel and has been used to manage malignant hypertensive crisis ${ }^{4}$ and palliate hyperfunctioning malignant tumours. 


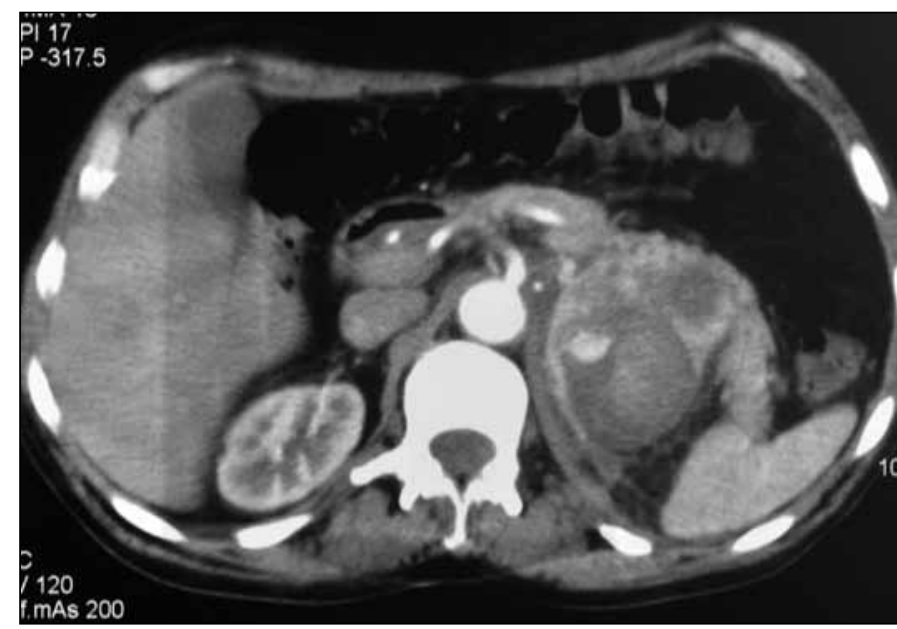

Fig. 1. A contrast enhanced computed tomography showing the retroperitoneal heamorrhage left suprarenal retroperitoneum.

In cases of retroperitoneal hemorrhage presenting with hemodynamic instability, TAE can be an effective and a safe procedure for achieving hemostasis and maintaining the patient in optimal condition until surgery. A review of 53 cases of ruptured pheochromocytoma revealed that 17 (32\%) of the patients died, and that no mortality occurred among the 12 patients who received an alpha blocker (for high blood pressure) and fluid infusion therapy before surgery. Only 3 patients underwent elective surgery after TAE with

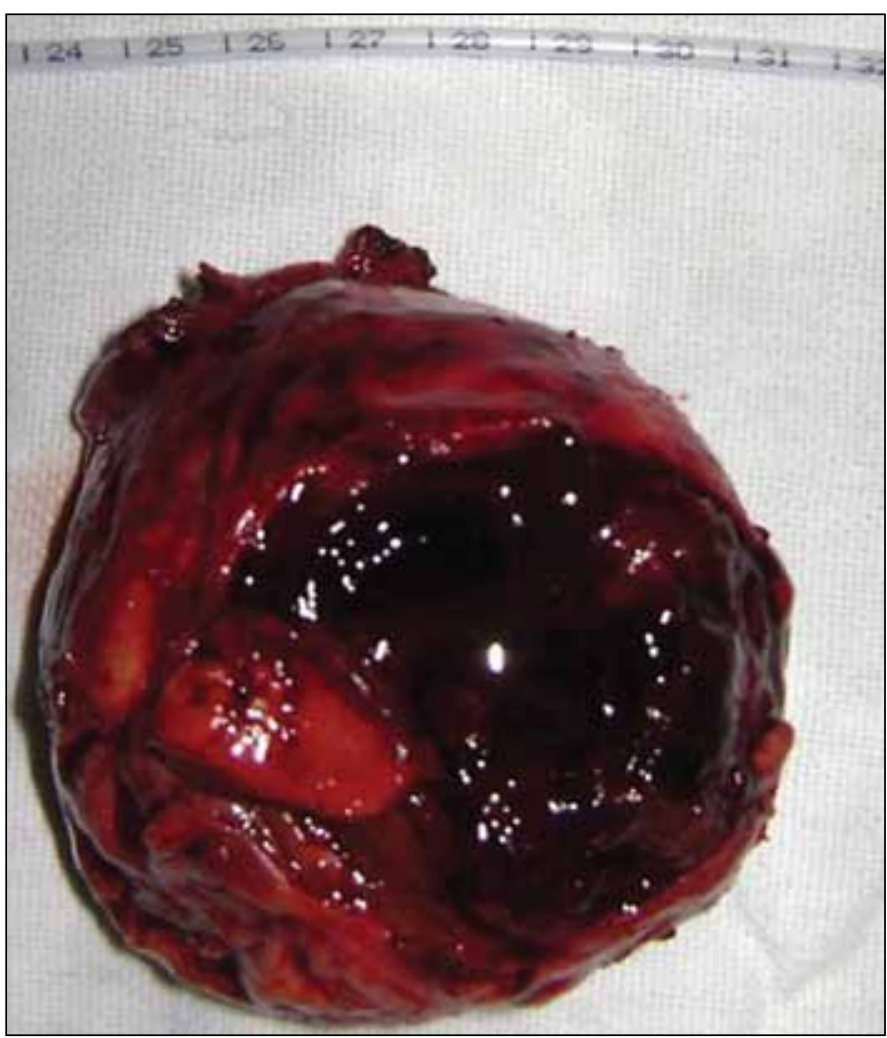

Fig 3a. Excised tumour with ruptured capsule with hematoma.

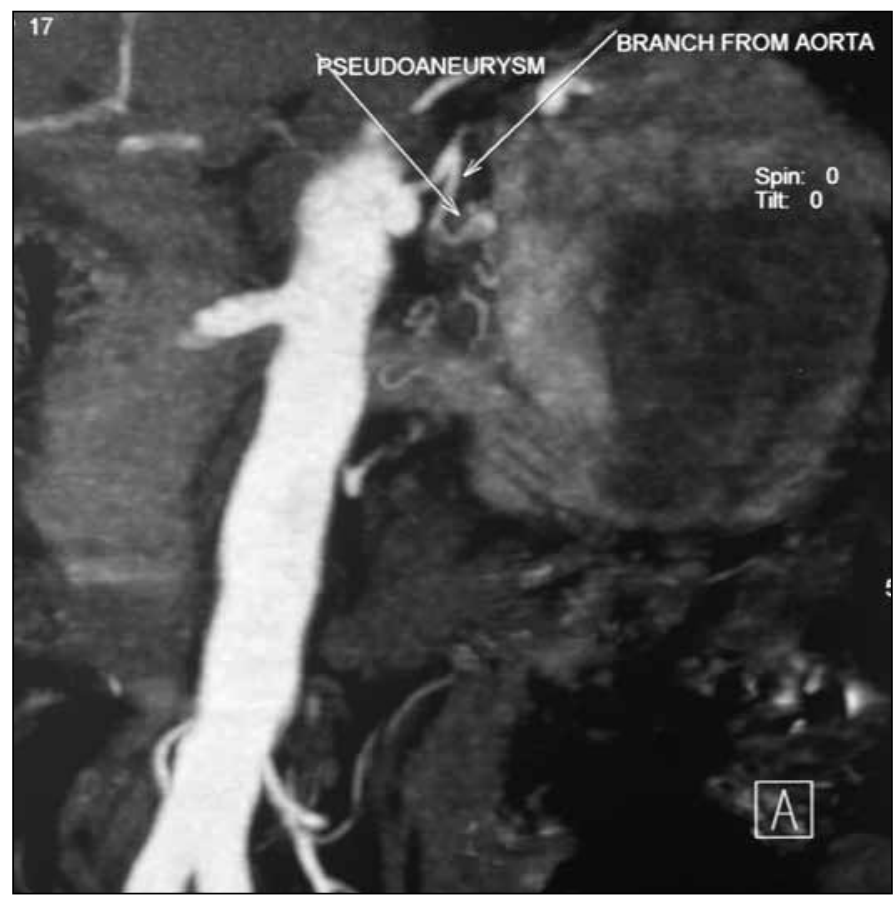

Fig. 2. Coronal section contrast enhanced computed tomography image showing left adrenal artery pseudoaneurysm.

no reported mortality. ${ }^{5}$ This emphasizes the fact that these patients merit resuscitation and stabilization before deciding to operate. Even in situations wherein the workup for pheochromocytoma remains negative (as in conditions with infarcted pheochromocytoma), all necessary precautions for pheochromocytoma are to be taken during the preparation.

During initial resuscitation of these patients, two pathophysiological processes have to be addressed simultaneously. Massive release of catecholamines can lead to hypertensive crisis and ongoing blood loss can lead to hypotension.

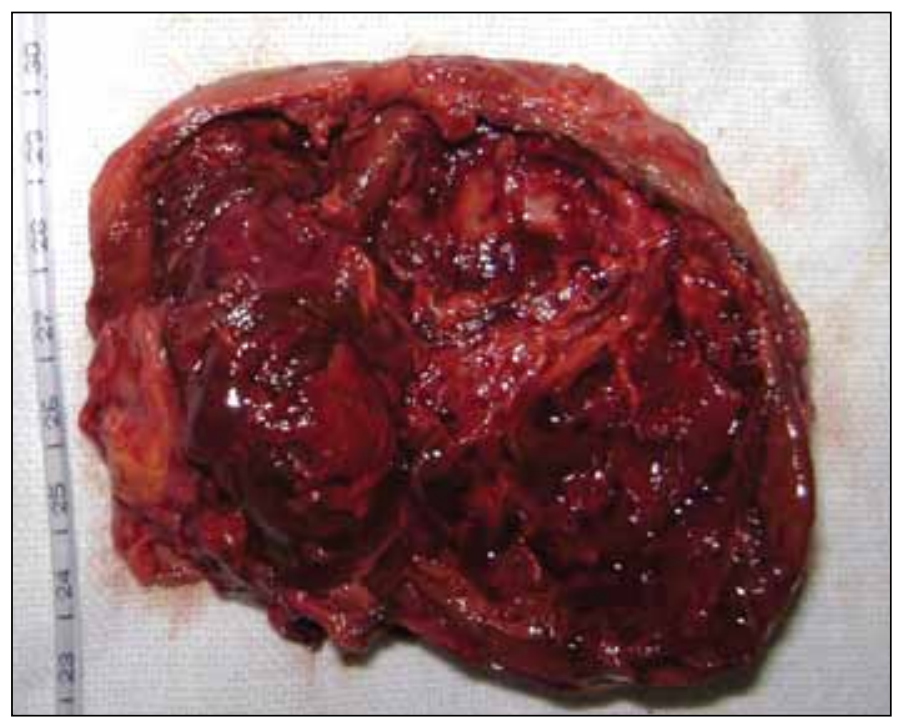

Fig 3b. Cutsection of specimen. 


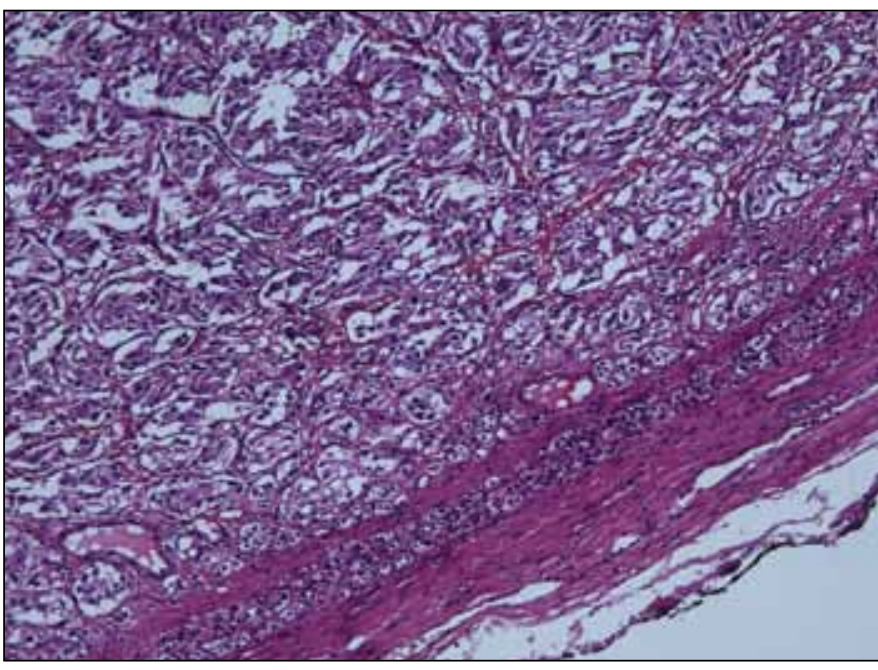

Fig 4a. Photomicrograph showing tumour with compressed rim of normal adrenal cortex on the top (hematoxylin \& eosin, $\times 40$ ).

Therefore, once the blood loss is controlled with TAE, the catecholamines regain their impact and can cause hypertensive crisis or fluctuating blood pressure. Once initial hemodynamic stability is obtained, it should be sustained by careful titration of the antihypertensive infusions and fluid replacement, under close patient monitoring.

\section{Conclusion}

In this case, rupture of pheochromocytoma and adrenal artery were probably due to increased intra-tumoral intravascular pressure which may be precipitated by paroxysms of hypertension or necrosis. Prompt use of cross-sectional imaging and clinical suspicion will help to recognize the correct pathology and will allow physicians to treat patients expeditiously. Suspicion of pheochromocytoma in a patient presenting with hypotension is unlikely, but still has to be considered. Our case, albeit a rare clinical presentation, heightens our awareness of the clinical paradox.

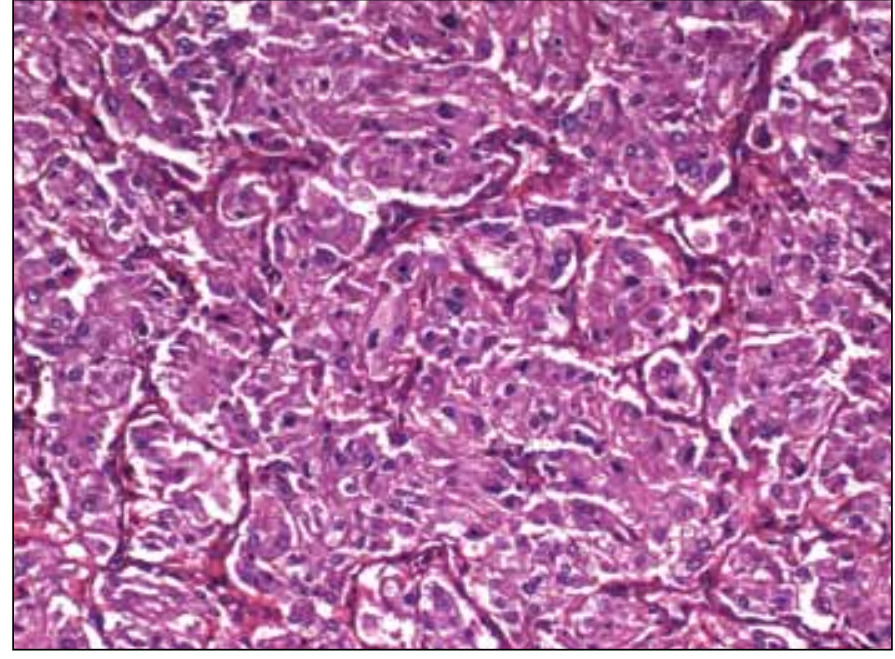

Fig 4b. Photomicrograph showing typical growth pattern of nests of tumour cells (zellballen) surrounded by fibrovascular stroma (hematoxylin \& eosin, $\times 200$ ).

Competing interests: None dedared.

This paper has been peer-reviewed.

\section{References}

1. Kobayashi T, Iwai A, Takahashi R, et al. Spontaneous rupture of adrenal pheochromocytoma: review and analysis of prognostic factors. J Surg Oncol 2005;90:31-5. http://dx.doi.org/10.1002/iso.20234

2. Keiser HR. Pheochromocytoma and related tumors. Leslie J, DeGroot J, Larry Jameson, editors. Endocrinology. Volume 3. Philadelphia, PA: Saunders, 1995:1862-83.

3. Tanaka K, Noguchi S, Shuin T, et al. Spontaneous rupture of adrenal pheochromocytoma: a case report. J Urol 1994:151:120-1.

4. Hrabovsky EE, Mclellan D, Horton JA, et al. Catheter embolization: preparation of patient with pheochromocytoma. J Pediatr Surg 1982;17:849-50. http://dx.doi.org/10.1016/S0022-3468(82)80455-7

5. Habib M, Tarazi I, Batta M. Arterial embolization for ruptured adrenal pheochromocytoma. Curr Oncol 2010;17:65-70. http://dx.doi.org/10.3747/co.v17i6.597

Correspondence: Dr. Santosh Kumar, Assistant Professor, Department of Urology, PGIMER, Chandigarh; fax: 91-172-2744401; santoshsp1967jaimatadi@yahoo.co.in 